\title{
UNE MÉTHODE DE PRODUCTION DE TRUITES FARIO DESTINÉES AUX REPEUPLEMENTS DE RESTAURATION
}

\author{
G. MAISSE *, J.P. PORCHER *
}

\section{RESUME}

Une méthode de production de populations de truites fario présentant une forte variabilité génétique est proposee pour les repeuplements de restauration.

Du sperme de geniteurs sauvage est conservé à des temperatures voisines de 0 'C et à l'obscurité, pendant la durée du transport entre le cours d'eau d'origine et la pisciculture, où l'on procède à l'insémination d'ovules de truites fario d'élevage en utilisant le dilueur de sperme 532.

La descendance est destinée de preference aux déversements au stade œuf dans le milieu préalablement restauré.

- IN.R.A., Laboratoire de Physiologie des Poissons. Campus de Rennes Beaulieu - 35042 RENNES Cedex

* C.S.P., Délégation régionale de Rennes. 14, rue Massenet - 35100 RENNES.

Article available at http://www.kmae-journal.org or http://dx.doi.org/10.1051/kmae:1981033 


\section{INTRODUCTION}

Nous rapallerons ICI quavant de decider une operation de repeuplement en salmonidés, il est nécessaire de définir la capacité d'accueil du milieu à repeupler et d'évaluer la population en place et son taux de renouvellement (CUINAT, 1971; CHE. VASSUS, 1978).

Bien souvent, des operations d'aménagements du milieu (suppression des points Je pollution, installation de passes à poissons, nettoyage des zones de frayère et de production) suffiraient à maintenir un stock de géniteurs compatible avec une pression de pêche. Dans tous les cas, un repeuplement dans un milieu dégradé est réalisé en pure perte (PIERRE, THIBAULT, 1974).

Cet article se place dans le cas où l'on décide de peupler un milieu neuf (lac de montagne par exemple) ou de repeupler un milieu ayant subi une pollution, chronique ou accidentelle, conduisant à un anéantissement quasi total du cheptel de salmonidés Dans ces Jeux cas, on fait en outre l'hypothèse que la reproduction des salmonidés est possible dans le milieu considere. C'est ce que CHEVASSUS (1978) appelle le repeuplement de restauration. Il est alors souhaitable de mettre en place une souche adaptee à l'environnement d'accueil.

En labsence de connaissances précises sur les caracteristiques génétiques que devra posseder une telle souche, une solution interessante est d'introduire une population dont les cazacités d'adajtation génétique à long terme, par le biais de la sélection naturelle sont importantes.

Un tel résultat sera obtenu par l'utilisation de populations très polymorphes, présentant une forte variabilité génétique et donc a priori "adaptables " à des milieux très divers (CHEVASSUS, 1978; GUYOMARD, 1978).

En prenant le cas de la Truite fario, espèce européenne indigène dont l'élevage est maiłrisé, nous proposons de conserver en pisciculture une ou plusieurs souches bien adaptèes aux conditions d'élevage, dont les femelles donneront les ovules qui seron: fécondés par du sperme collecté sur des mâles sauvages issus du plus grand nombre possible de bassins hydrographiques de la région. L'élevage des femelles est le moyen d'obtenir un grand nombre d'ovules et par conséquent d'individus de repeuplement sans opérer de ponctions dans les populations sauvages

Les collectes de spermes en milieu nature! introduiront la variabilité génétique souhaitée et éviteront le transport et le stockage de mâles sauvages, souvent difficiles en periode de reproduction.

\section{PRELEVEMENT DU SPERME SUR LES GENITEURS SAUVAGES ET SA CONSER- VATION}

Le prélèvement du sperme sur les geniteurs sauvages est décidé au moment ou les femelles de pisciculture sont mûres.

La conservation du sperme à des températures voisines de $0{ }^{\circ} \mathrm{C}$ et à l'obscurité se traduit après $24 \mathrm{~h}$, jar une chute de $25 \%$ du pouvoir fécondant (CARPENTIER et BILLARD, 1978).

STOSS et al. (1978) ont montre diune part que la conservation du sperme est zméliorée sous atmosphère d'oxygène et d'autre part que le mélange des spermes ne nult pas à leur conservation.

Ce sont les raisons pour lesquelles nous préconisons, pour des durées supérieures à $6 \mathrm{~h}$ et inférieures à $48 \mathrm{~h}$, de procéder à la conservation de mélanges de spermes en fable érasseur, sous atmosphère d’oxygène, dans un récipient maintenu dans la glace et à l'obscurité. 


\section{$1^{\circ}$. Matériel et personnel nécessaires}

- Un matériel de pêche électrique:

- 2 épuisettes à filet en nylon sans nœud;

- 2 seaux de 15 litres:

- 1 bac de 50 litres :

- 2 viviers:

- anesthésiant : Phénoxyéthanol ou MS 222 ;

- des serpillières:

- une glacière contenant de la glace pilee ;

- 30 tubes à essais en verre de $5 \mathrm{ml}$ avec bouchons;

- une dizaine de sacs en plastique (1 litre);

- une vingtaine de bracelets élastiques;

- une bouteille d'oxygène avec un dètendeur et un tuyau souple;

- 3 personnes.

\section{Déroulement des opérations}

a) Les mâles spermiants sont capturés par pêche électrique et stockés en vivier

b) Ils sont anesthésiés dans une solution de Phénoxyéthanol ( $3 \mathrm{ml}$ four 10 litres d'eau) (photo $n^{\circ} 1$ ).

Le Phénoxyethanol devient solide par basse temperature.

11 est alors conseillé d'utiliser un mélange contenant $2 / 3$ de Phénoxyéthanol et $1 / 3$ d'Ethanol qui reste liquide jusqu'à $-10^{\circ} \mathrm{C}$ et se dissout mieux dans l'eau.

La dose de mélange à utiliser est de $4.5 \mathrm{ml}$ pour 10 litres d'eau.

c) Le poisson est égoutté soigneusement dans une serpillière humide. II est imperatif de ne pas frotter les animaux pour ne pas enlever la couche de mucus pro. tecteur (photo $n^{\circ} 2$ ).

d) On procède au prèlèvement du sperme par pression des flancs de l'animal, après ślimination des fèces éventuelles et de l'urine (photo $n^{\circ} 3$ ). Le sperme est recue:lli dans un tube ( $1 \mathrm{ml}$ au maximum par tube) qui est immédiatement bouché puis placé dans la glace pilée, à l'obscurité. La présence avec le sperme d'une seule goutte d'eau entrainera le rejet de l'échantillon.

En prévision du transport de longue durée (superieure à 6 heures), il est conseilie de regrouper les differents spermes dans un ou plusie'urs sacs en plastique gonflés à l'oxygène, hermétiquement clos, puis déposés dans la glacière à l'obscuritè (méthode comparable à celle du transport des alevins) (photo $n^{\circ} 4$ ).

e) Les mâles donneurs sont mis à récupérer dans un vivier puis remis en liberté dans le ruissea'

f) Compte tenu des possibilités de conservation, il est possible de réaliser dans la mème journée plusieurs collectes en des points differen's du bassin hydrographique. 


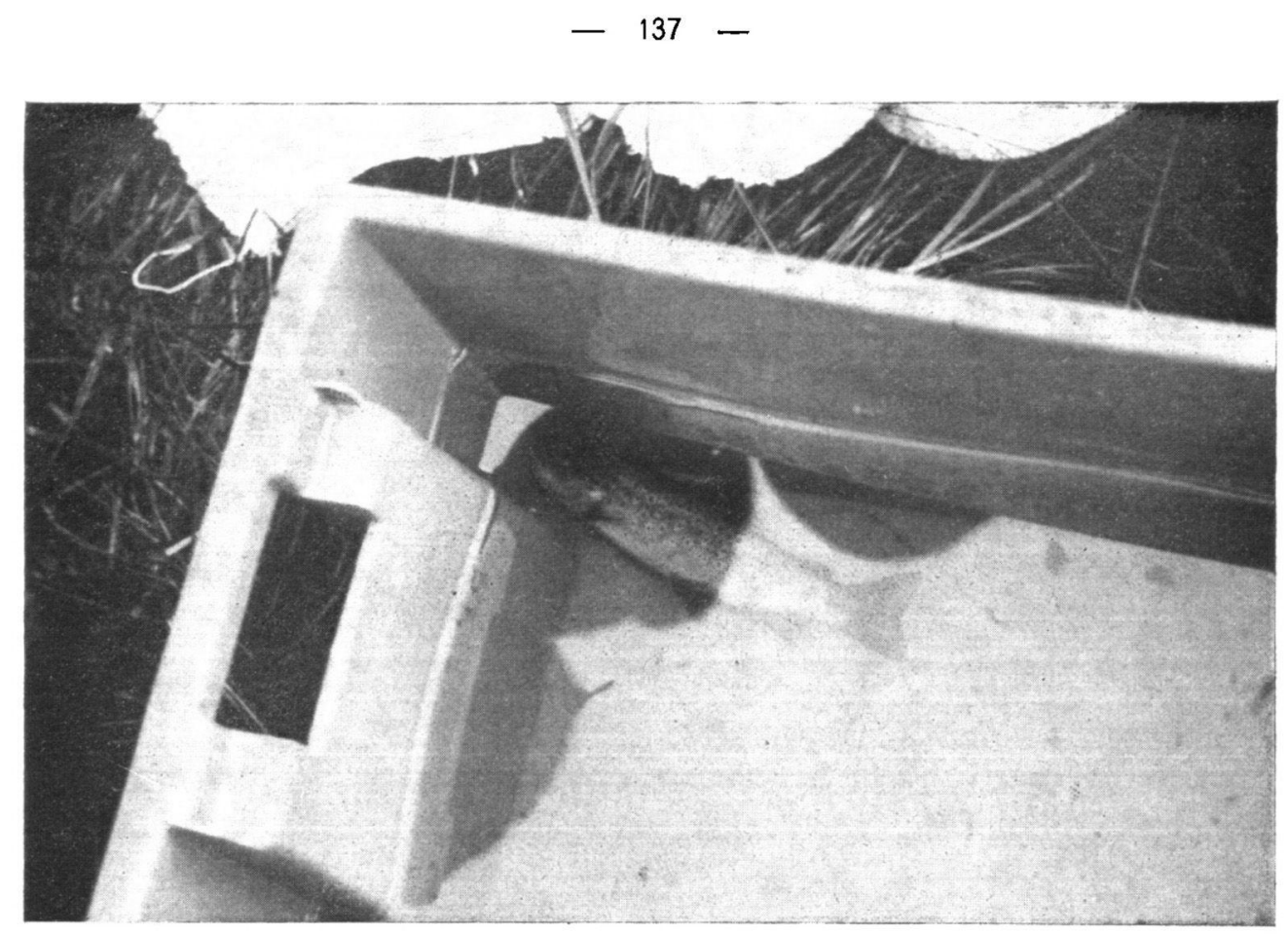

Photo $n^{\circ} 1$ : Anesthésie des máles au phénoxyéthanol.

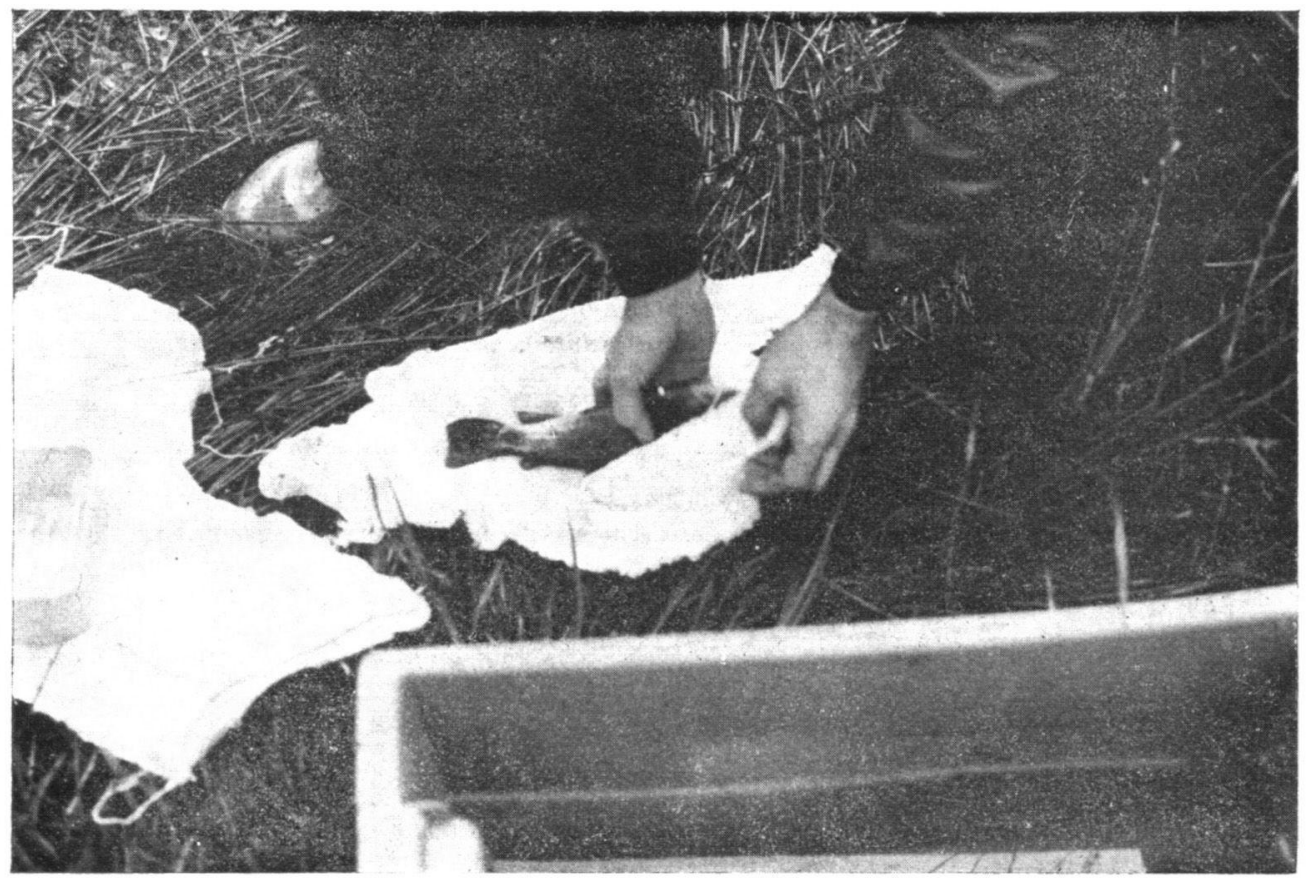

Photo $n^{\circ} 2$ : Egouttage des poissons dans une serpillière humide. 


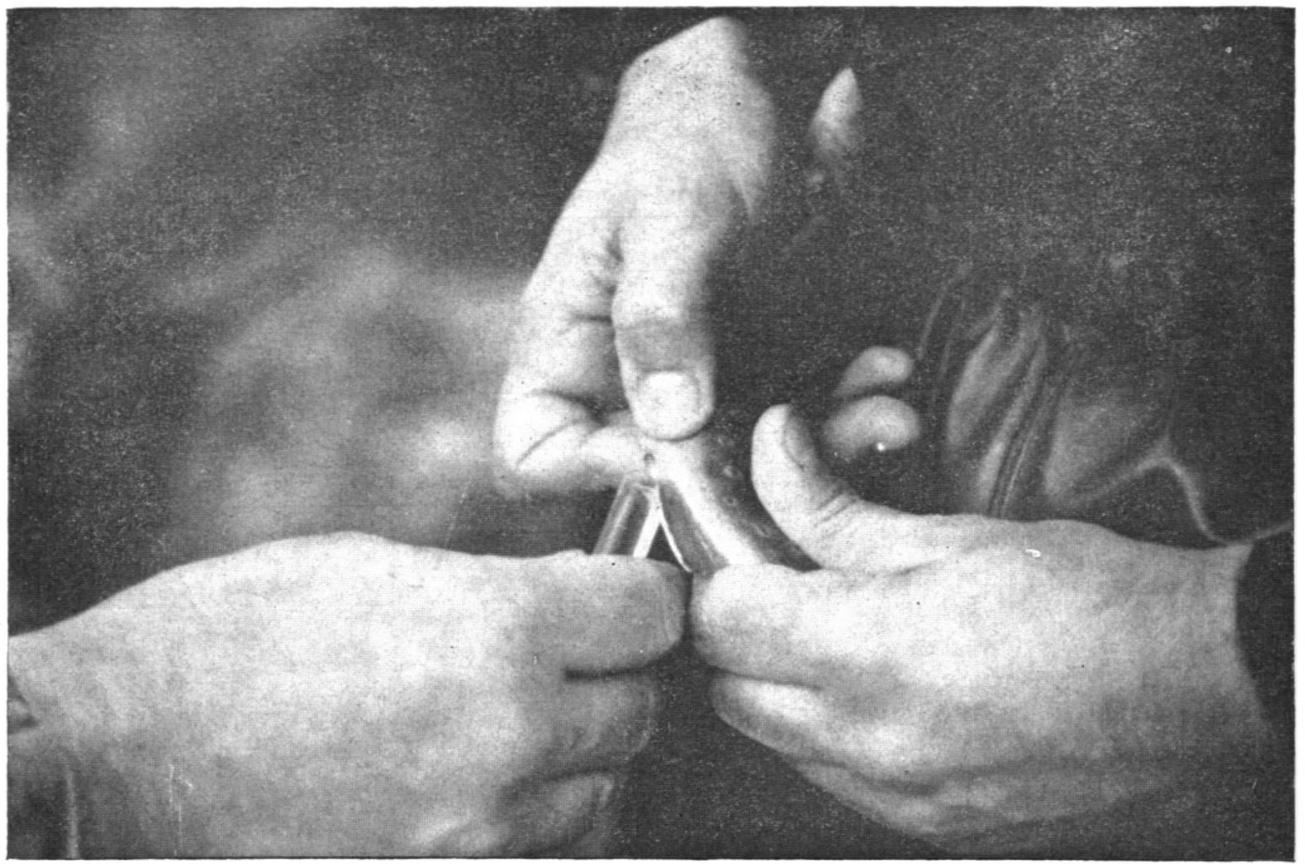

Photo $n^{\circ} 3$ : Prélèvement du sperme.

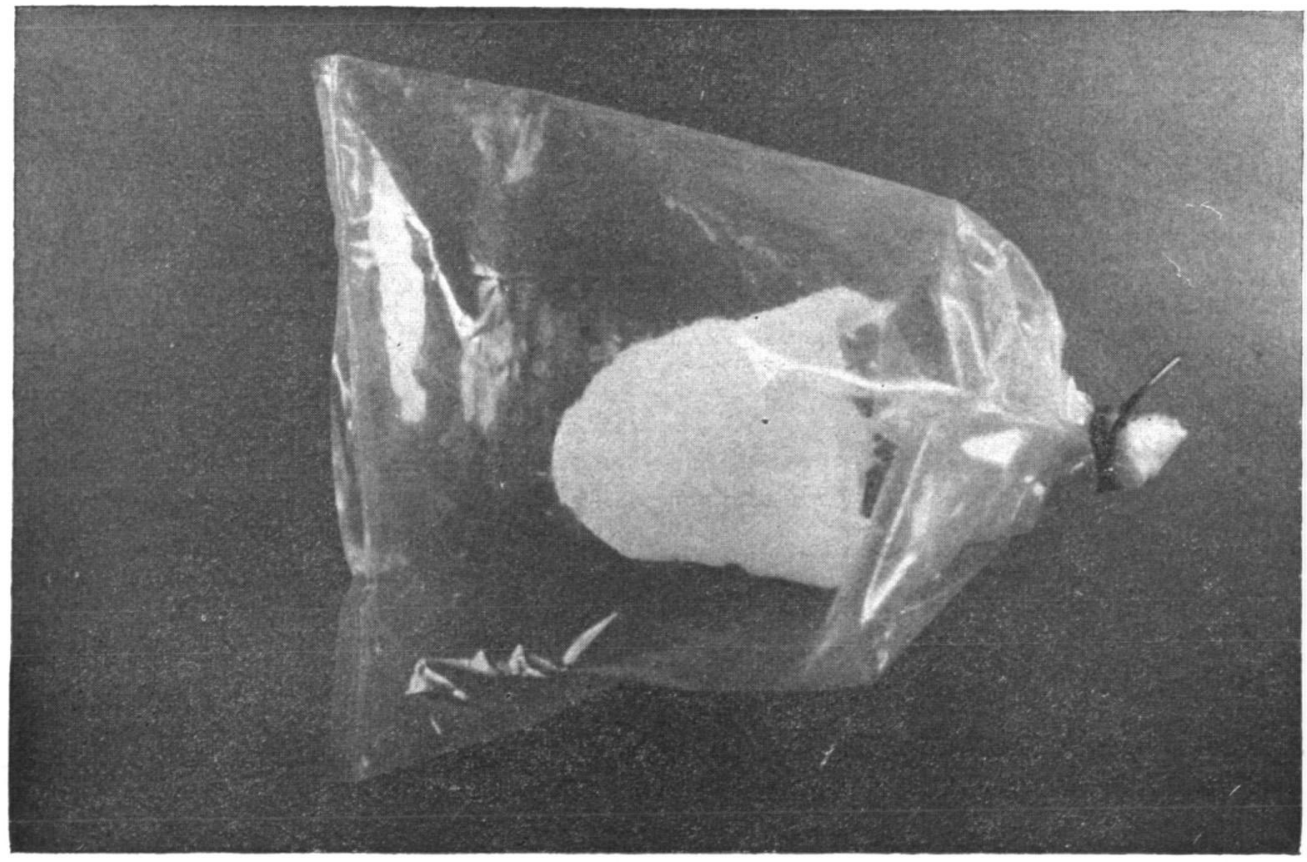

Photo $n^{\circ} 4$ : Sac plastique gonflé à l'oxygène contenant un mélange de spermes (vue de dessus). 


\section{FECONDATION A LA PISCICULTURE}

Nous avons constaté que les femelles d'élevage étaient en pleine période d'ovulation au moment où les mâles sauvages ètaient en début de spermiation, ce qui se traduit généralement par une faible production d'un sperme de médiocre qualité (BILLARD. 1979). C'est pourquoi l'utilisation du difueur d'insémination (PETIT et al. 1973; BILLARD et al. 1974: BILLARD 1977 a) est indispensable pour obtenir une bonne fécondation.

La technique d'insémination artificielle est celle décrite par BILLARD (1977b).

\section{$1^{\circ}$. Matériel et personnel nécessaires}

- 1 épuisette à filet en nylon sans nœud:

- 1 bac de 50 litres:

- anesthésiant : Phénoxyéthanol ou MS 222;

- 1 table ;

- des serpillières:

- une passoire:

- une dizaine de boites en plastique ( 1 litre);

- une pipette graduée de $5 \mathrm{ml}$ :

- une bouteille en plastique (1 litre) à bouchon à vis ;

- dilueur 532 - Laboratoire LATHEVET - distribué par la Cooperative nationale agricole de la Pisciculture, 11, rue Milton - 75009 PARIS;

- si possible un microscope $(G \times 100)$ et des lames porte-objet ;

- 3 personnes.

\section{$2^{\circ}$. Déroulement des opérations}

a) Dans la mesure du possible, contrôler la motilité du sperme au microscope (au grossissement 100) sur une lame en présence d'une goutte d'eau au contact de laquelle les spermatozoides vivants se mettent en mouvement avant de s'immobiliser au bout d'une trentaine de secondes.

b) On procède au mélange de plusieurs spermes, si cela n'a pas èté fait au bord du ruisseau.

c) Les femelles ovulèes, à jeun $\left(48 \mathrm{~h}\right.$ à $\left.10{ }^{\circ} \mathrm{C}\right)$ sont anesthésiées dans une solution de Phénoxyéthanol ( $3 \mathrm{ml}$ pour 10 litres d'eau), puis égouttées sur une serpillière humide.

d) L'expulsion des ovules est obtenue par pression des flancs. Les ovules sont recueillis dans une passoire, afin d'éliminer le liquide ovarien, puis transférés dans une boite en plastique sèche (photo $n^{\circ} 5$ ).

e) Le sperme est déposé sur les ovules à raison de $1 \mathrm{ml}$ par litre d'ovules (photo $n^{\circ}$ 6).

f) On ajoute 0.5 I de dilueur d'insémination (532) par litre d'ovules (photo $n^{\circ} 7$ )

g) On mélange le tout par transvasements répétés dans une autre boite (photo $n^{\circ} 8$ )

h) Après les avoir laissés reposer 15 minutes, les œufs sont lavés puis mis dans les incubateurs. 


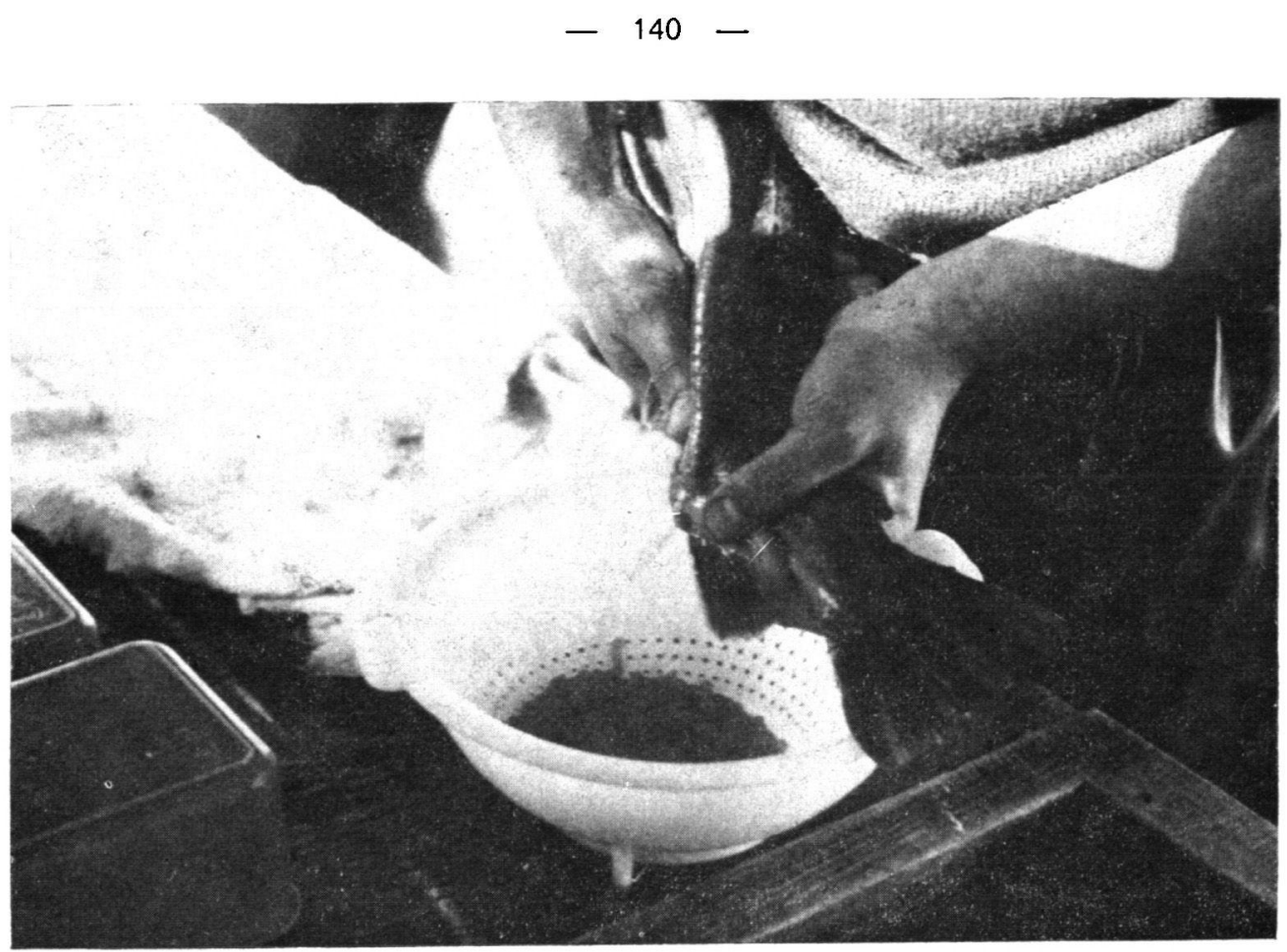

Photo $n^{\circ} 5$ : Prélèvement des ovules.

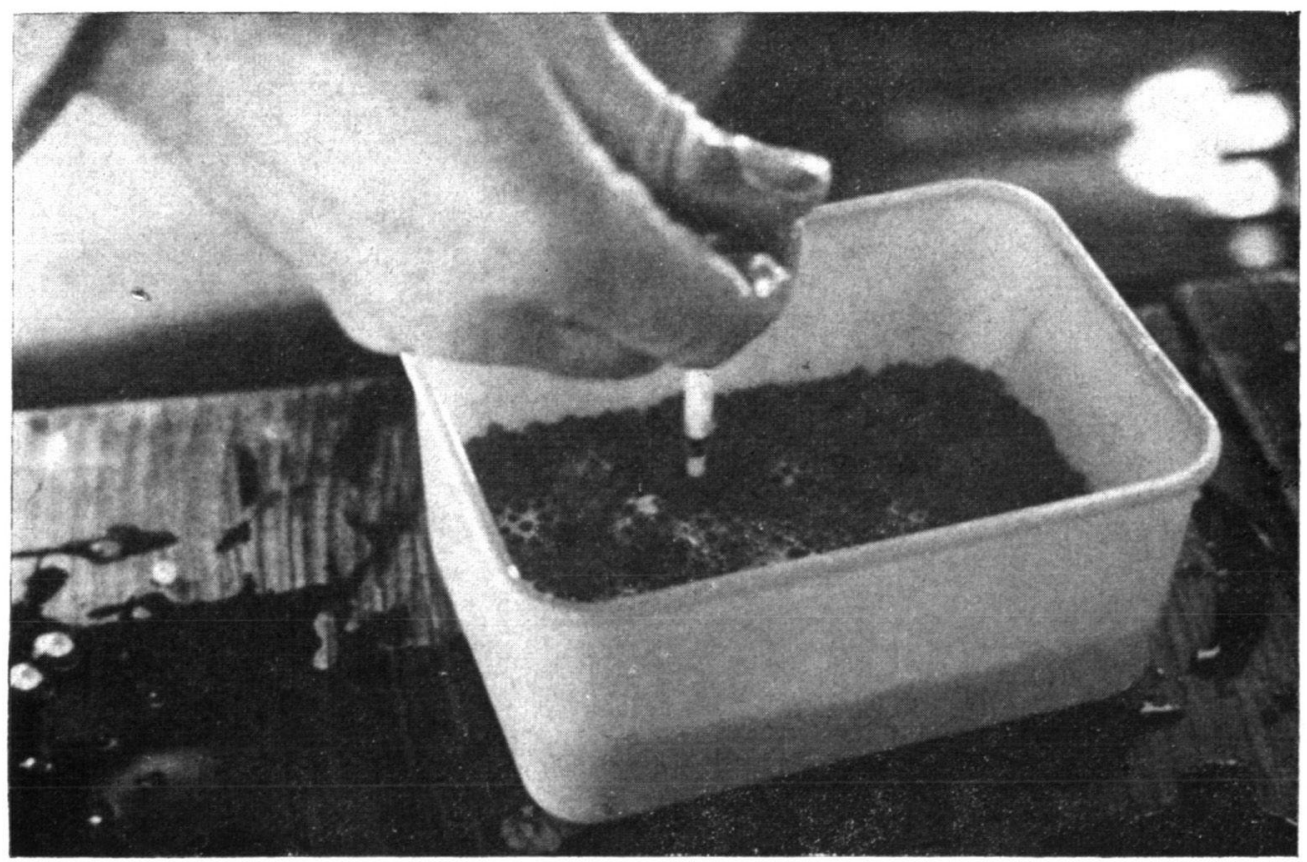

Photo $n^{\circ} 6$ : Dépót du sperme sur les ovules. 


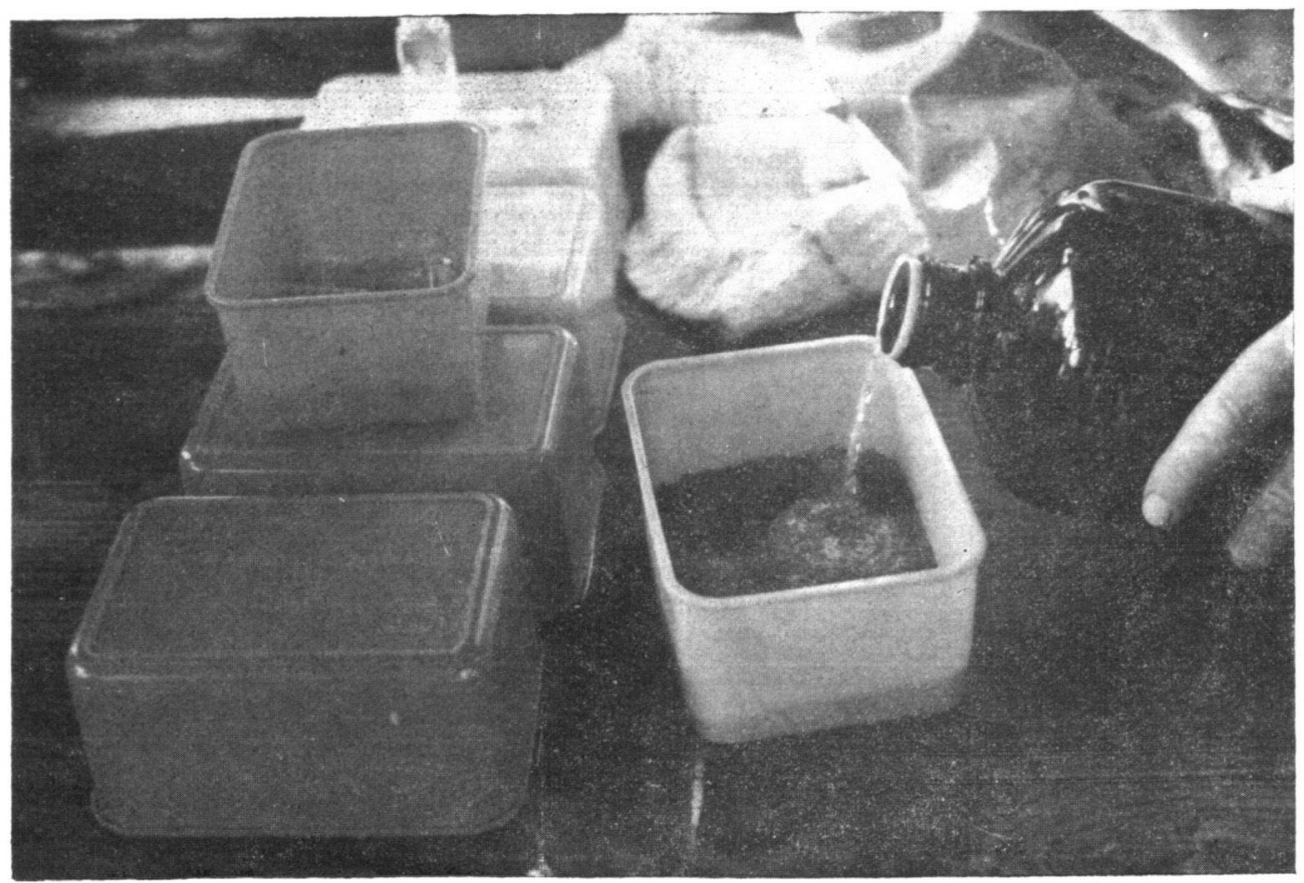

Photo $n^{\circ} 7$ : Addition de dilueur.

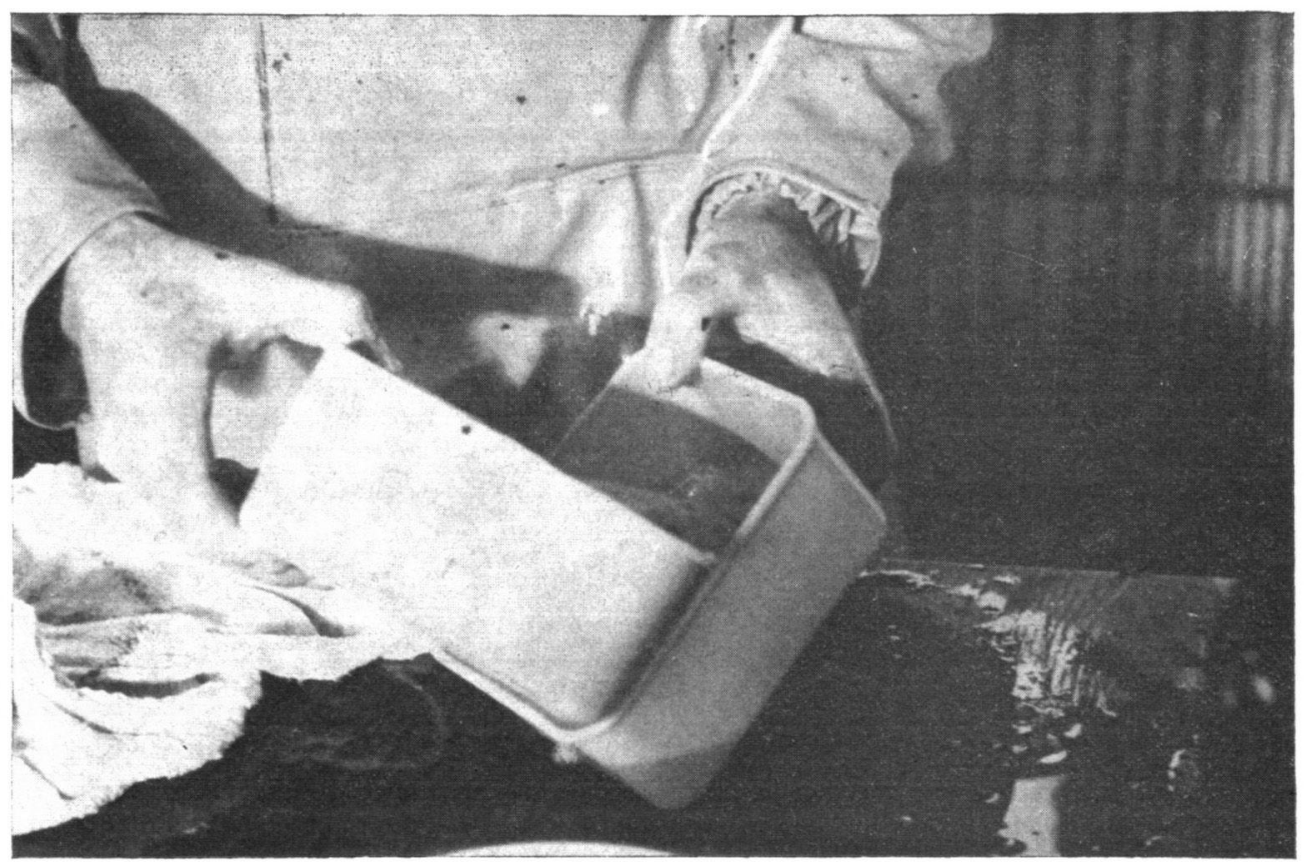

Photo $n^{\circ} 8$ : Mèlange par transvasement des gamètes et du dilueur. 


\section{RESULTATS ENREGISTRES EN 1979}

En novembre 1979, cette methode a èté testee deux fois à la pisciculture interfederale de la Gouarnais (Morbihan) sur des truites fario.

Dans un premier cas. le sperme a èté prélevé sur des mâles sauvages d'un ruisseau situé à quelques kilomètres de la pisciculture et conservé penjant cinq heures avant la fécondation.

Chaque ponte de 16 femelles d'élevage a été séparée en deux lots qui ont été insemines par du sperme provenant soit des mâles sauvages, soit des mâles d'élevago. Dans un premier cas, l'insémination a été pratiquée suivant le protocole décrit ci-dessus: dans le second cas, la méthode employée est celle qui est utilisée traditionnellement dans la pisciculture, sans utilisation de dilueur.

Le pourcentage de réussite observé au stade œillé a été de $92 \%$ pour les semisauvages et de $93 \%$ pour les produits d'élevage. Dans les conditions d'incubation on ne peut pas affirmer que cette différence soit significative. Le deuxième test a èté réalisé avac du sperme prélevé sur des géniteurs sauvages du bassin du Couesnon (Ille et Vilaine).

La fécondation a eu lieu $18 \mathrm{~h}$ après à la Gouarnais avec utilisation du dilueur. Le taux de réussite au stade œillé a été de $92 \%$ et le taux. d'éclosion de $90 \%$ (voir tableau $n^{\circ}$ 1).

\begin{tabular}{|l|r|r|r|}
\hline & Test $n^{\circ} 1$ & Test $n^{\circ} 2$ \\
\hline Nombrc total d'œufs & Lot élevage & Lot semi-sauvage \\
Nombre d'œufs œillés & 18000 & 18000 & 20000 \\
\hline de réussite & 16560 & 17280 & 18400 \\
\hline
\end{tabular}

Tableau $n^{\circ} 1$ : Résultats des deux tests.

\section{DISCUSSION - CONCLUSION}

Compte tenu du risque d'appauvrissement du potentiel de reproduction des cours d'eau où l'on capturerait un grand nombre te géniteurs destinés à produire des individus de repeuplement (VIBERT et LAGLER, 1961) CUINAT (1971) propose de capturer uniquement des mâles, de les transporter à la pisciculture, où l'insémination sera réalisée. puis de les remettre dans le milieu naturel.

Cette méthode. qui présente l'avantage de protéger les femelles, laisse peu de chancs aux mâles sauvages utilisés de participer par la suite à la fraie naturelle, voire de survivre aux conditions de pisciculture, même temporaires.

La méthode, décrite Jans cet article, utilise des techniques simples (prélèvement des gamètes à sec, conservation du sperme à des températures voisines de $0{ }^{\circ} \mathrm{C}$. utilisation du dilueur d'insémination\} qui permettent d'apporter une réponse au problème de la sauvegarde des géniteurs sauvages. Il n'est pas exclu que les mâles sauvages capturés pour la collecte du sperme pourront participer à la fraie naturelle à condition 
que les plus grands soins soient apportés à leur manipulation, leur physiologie en période de reproduction les rendant particulièrement sensibles aux maladies cutanées (ROBERT. 1978).

La pisciculture de production d'ovules devra être inscrite au contôle sanitaire. Le cheptel de femelles devra possèder une bonne aptitude aux conditions d'élevage et une bonne fécondité (réforme des femelles âgèes de plus de 4 ans).

Les femelles donnant des gros œufs seront recherchees, les alevins, qui en sont issus, presentant une meilleure survie que ceux produits par de petits œufs (BAGENAL, 1969)

La production de populations " $100 \%$ femelles" par la technique des lignées gynogénétiques (CHEVASSUS et al., 1979) pourra permettre d'élever dans chaque région un cheptel de femelles, destiné à répondre à toute destruction de géniteurs sauvages provoquée par une pollution aigüe ou chronique, en utilisant la méthode décrite ci-dessus.

La congélation du sperme de salmonidés (STEIN et BAYRLE, 1978 ; STOSS et al., 1978; ZELL, 1978) n'est pas encore une technique suffisamment fiable pour créer de véritables banques de sperme associées à ces élevages de femelles.

Compte tenu de l'objectif de restauration d'une population de truites, il est souhaitable de procéder à un déversement précoce des individus produits, en accord avec CUINAT (1971) qui signale que des repeuplements réalisés aux stades œuf ou a'evin vésiculé Jonnent lieu à une implantation des animaux déversés a généralement analogue à celle des sujets indigènes.

Les déversements au stade œuf s'effectueront à l'aide de boites Vibert (VIBERT, 1977). Les cufs seront desinfectés aux iodophores (GERARD, 1974) par trempage des boites pleines dans la solution désinfectante. juste avant le déversement.

Les alevins vésiculés ne devront provenir que d'une pisciculture reconnue saine

Dans le cas où l'on sera obligé de procéder à un repeuplement au stałe truitelle, :I est conseillé de pratiquer l'élevage, sous contrôle sanitaire, en rigole d'alevinage en semi-intensif avec apport de nourriture artificielle (CUINAT et DUMAS, 1974).

\section{REMERCIEMENTS}

Nous remercions particulièrement MM. KERDAL et GUILLERME, pisciculteurs à la Gouarnais, ainsi que la Fédération Départementale des Associations de Pêche et de Pisciculture et la Garderie commissionnée du Morbihan qui ont apporté leur concours à cette expérimentation.

\section{B!BLIOGRAPHIE}

BAGENAL T.B., 1969. Relationship between egg size and fry survival in brown trout Salmo trutta L. J. Fish. Biol. 1, 4, 319-353

BILLARD R., 1977 a. Utilisation d'un systeme Tris-Glycocolle pour tamponner le dilueur d'insemination pour truite. Bull. Fr. Piscic. 264, 102-112.

BILLARD R. 1977 b. A new technique of artificial insemination for salmonids using a sperm diluent. Fisheries, 1, 24-25

BILLARD R., 1979. La gamétogenèse, le cycle sexuel et le contrôle de la reproduction chez les poissons téléostéens. Bull. Fr. Piscic. 273. 117-136. 
BILLARD R., JALABERT B., et BRETON B. 1974. L'insèmination artificielle de la truite. III. Définition de la nature et de la molarité du tampon à employer avec les dilueurs d'insémination et de conservation. Ann. Biol. Anim. Bioch. Biophys. 14 (4 - A) 611-621.

CARPENTIER P., et BILLARD R., 1978. Conservation à court terme des gamètes de salmonidés à des temperatures voisines de $0{ }^{\circ} \mathrm{C}$. Ann. Biol. Anim. Bioch. Biophys. 18 (4) $1083-1088$.

CHEVASSUS B., 1978. Quelques réflexions sur la notion de * Génotype adapte au repeuplement. Document de travail pour la deuxième réunion du GGPEA, $7 \mathrm{p}$

CHEVASSUS B., CHOURROUT D., JALABERT B., 1979. Le contrôle de la reproduction chez les poissons. I. Les populations - monosexes. Bull. Fr. Piscic. 274, 18-31

CUINAT R., 1971. Ecologie et repeuplement des cours d'eau à truite. Bull. Fr. Piscic. 240-242-243.

CUINAT R. et DUMAS 1., 1974. Elevage semi-intensif de truitelles de repeuplement. Bull. Fr. Piscic. 252, 93-104.

GERARD J.P., 1974. Note technique $n^{\circ} 03$ sur l'emploi des iodophores en pisciculture. Bull. Fr. Piscic. 254, 13-15.

GUYOMARD R., 1978. Génétique biochimique et gestion des populations naturelles Bulletin scientifique et technique - département d'hydrobiologie - I.N.R.A. - 18 p.

PETIT J., JALABERT B., CHEVASSUS B. et BILLARD R., 1973. L'insémination artificielle de la Truite (Salmo gairdneri Richardson). I. Effet du taux de dilution, du $\mathrm{pH}$ et de la pression osmotique du dilueur sur la fécondation. Ann. Hydrobiol. 4 (2) 201-210.

PIERRE J.C. et THIBAULT M., 1974. Le repeuplement du Scorff en Saumon atlantique (Salmo salar L. 1758). Le point sur les travaux entrepris. Bull. Fr. Piscic. 252, 105-117.

ROBERTS R.J., 1978. Pathologie du poisson. Traduction française de P. d'AUTHEVILLE. Maloine S.A. éditeur, 1 Vol. 317 p.

STEIN H. et BAYRLE H., 1978. Cryopreservation of the sperm of some freshwater teleosts. Ann. Biol. Anim. Bioch. Biophys. 18 (4) 1073-1076.

STOSS J., BUYUKHATIPOGLU S., HOLTZ W., 1978. Short term in cryopreservation of rainbow trout (Salmo gairdneri Richardson) sperm. Ann. Biol. Anim. Bioch. Biophys. 18 (4) $1077-1082$.

VIBERT R., 1977. Boite Vibert : nouvelles précisions sur leur utilisation et leurs résultats. Piscic. Franç. 50, 24-32.

VIBERT R., LAGLER K.F., 1961. Pèche continentale, Biologie et Aménagement. Paris Dunod, 1 Vol. 720 p.

ZELL S.R., 1978. Cryopreservation of gametes and embryos of salmonid fishes. Ann. Biol. Anim. Bioch. Biophys 18 (4) 1089-1099 